\title{
On the constant congruence speed of tetration Marco Ripà
}

\author{
sPIqr Society, World Intelligence Network \\ Rome, Italy \\ e-mail: marcokrt1984@yahoo.it
}

\section{Received: 11 September $2019 \quad$ Revised: 11 August $2020 \quad$ Accepted: 13 August 2020}

\begin{abstract}
Integer tetration, the iterated exponentiation ${ }^{b} a$ for $a \in \mathbb{N}-\{0,1\}$, is characterized by fascinating periodicity properties involving its rightmost figures, in any numeral system. Taking into account a radix-10 number system, in the book "La strana coda della serie $n^{\wedge} n^{\wedge} \ldots{ }^{\wedge} n "(2011)$, the author analyzed how many new stable digits are generated by every unitary increment of the hyperexponent $b$, and he indicated this value as $V(a)$ or "congruence speed" of $a \not \equiv 0(\bmod 10)$. A few conjectures about $V(a)$ arose. If $b$ is sufficiently large, the congruence speed does not depend on $b$, taking on a (strictly positive) unique value. We derive the formula that describes $V(a)$ for every $a$ ending in 5. Moreover, we claim that $V(a)=1$ for any $a(\bmod 25) \in\{2,3,4,6,8,9,11,12,13,14,16,17,19,21,22,23\}$ and $V(a) \geq 2$ otherwise. Finally, we show the size of the fundamental period $\mathcal{P}$ for any of the remaining values of the congruence speed: if $V(a) \geq 2$, then $\mathcal{P}(V(a))=10^{V(a)+1}$.
\end{abstract}

Keywords: Number theory, Power tower, Tetration, Hyperoperation, Charmichael function, Euler's totient function, Primitive root, Exponentiation, Integer sequence, Congruence speed, Modular arithmetic, Stable digit, Rightmost digit, Cycle, Periodicity.

2010 Mathematics Subject Classification: 11A07, 11F33.

\section{Introduction}

In the present paper, we study recurrence properties involving the rightmost digits of the tetration ${ }^{b} a=a^{a^{a \cdots}}$ (b-times) [6], observing that, when the hyperexponent $b \in \mathbb{N}$ is sufficiently large and $a(\bmod 25) \not \equiv\{0,1,5,7,10,15,18,20,24\}$, the number of new stable digits generated by any unitary increment of $b$ is unitary as well: it depends only on the congruence modulo 25 of the base $a \in \mathbb{N}[9]$.

In Sections 4 and 5, we extend the aforementioned relation to the remaining values of $a$.

These new results would contribute to improve big numbers rightmost digits calculations, opening new scenarios in cryptography/cryptanalysis, too [13]. 


\section{Congruence speed of $a(\bmod 25)$}

It is well known that, for any arbitrarily large $n,{ }^{b} a$ originates a string of $n$ stable figures [10], thus we can say that ${ }^{b} a$ is well-defined modulo $10^{n}$, for any $b \geq b^{\prime}(n, a)[9,11,12]$.

It is possible to observe the same peculiarity considering many different numeral systems [4], but we only take into account the decimal one (radix-10).

Let us now introduce the definition of "congruence speed" as it was originally presented by Ripà in his book about the righmost digits of ${ }^{b} a[6]$.

Definition 1. Let $a \in \mathbb{N}-\{1\}$ be an arbitrary base which is not a multiple of 10 and let $b \in \mathbb{N}-\{0,1\}$ be such that ${ }^{b-1} a \equiv{ }^{b} a\left(\bmod 10^{d}\right) \wedge{ }^{b-1} a \not \equiv{ }^{b} a\left(\bmod 10^{d+1}\right)$, where $d \in \mathbb{N}$. We define $V(a, b)$ to be the non-negative integer such that

$$
{ }^{b} a \equiv{ }^{b+1} a\left(\bmod 10^{d+V(a)}\right) \wedge{ }^{b} a \not{ }^{b+1} a\left(\bmod 10^{d+V(a)+1}\right) .
$$

For simplicity, from here on out, we refer to $V(a)$ as the congruence speed of the base $a \in \mathbb{N}: a \not \equiv 0(\bmod 10)$ of the tetration ${ }^{b} a$.

\section{Conjectures about the congruence speed}

In this section we present the conjectures and a few remarks to point out their main implications, specifying that $\varphi(n)$ indicates Euler's totient function (which counts the number of positive integers up to a given $n \in \mathbb{N}-\{0\}$ that are relatively prime to $n$ ), while $\lambda(n)$ repesents the Charmichael lambda function (the reduced totient function given by the smallest positive divisor of $\varphi(n)$ that satisfies the conclusion of the well known Euler's totient theorem).

Before starting to discuss the main results, let us introduce a lemma that we will use later.

Lemma 1. Referring to Definition $1, \forall b \geq 2, V(3, b)=1$. If $b=1$, then $V(3, b)=0$.

Proof. If $b=1$, the lemma is trivially verified, $b=1 \Rightarrow 3 \not \equiv 3^{3}(\bmod 10) \Rightarrow 3 \not \equiv 7(\bmod 10)$.

In order to show that $b \geq 2 \Rightarrow V(3)=1$, we need to prove that

$$
\forall b \geq 2,{ }^{b} 3 \equiv{ }^{b+1} 3\left(\bmod 10^{b-1}\right) \wedge{ }^{b} 3 \not{ }^{b+1} 3\left(\bmod 10^{b}\right) ;
$$

and we start with the first congruence relation.

We prove by induction on $b$ that ${ }^{b} 3 \equiv{ }^{b+1} 3\left(\bmod 2^{b+1}\right), \forall b \geq 2$.

$$
b=2 \Rightarrow 27 \equiv 3^{27}(\bmod 8) \Rightarrow 3 \equiv 3(\bmod 8) \text {. }
$$

Let $b \geq 2$ and assume ${ }^{b} 3 \equiv{ }^{b+1} 3\left(\bmod 2^{b+1}\right)$. Since 3 and 10 are coprime, we can invoke Euler's totient theorem. It is well known that, $\forall b \in \mathbb{N}, \varphi\left(2^{b+2}\right)=2^{b+1}$ (proof follows from Euclid's lemma). Thus, $\left.3^{\left({ }^{b}\right)} \equiv 3^{\left({ }^{b+1} 3\right.}\right)\left(\bmod \varphi\left(2 \cdot 2^{b+2}\right)\right)$, and we can rewrite it as ${ }^{b+1} 3 \equiv{ }^{b+2} 3\left(\bmod 2^{b+2}\right)$, which concludes the proof of the inductive step.

Similarly, we can show that $\left.5^{b-1}\right|^{b+1} 3-{ }^{b} 3, \forall b \geq 2$.

Base case: $b=2 \Rightarrow 27 \equiv 3^{27}(\bmod 5) \Rightarrow 2 \equiv 2(\bmod 5)$.

Induction step: let $b \geq 2$ and assume ${ }^{b} 3 \equiv{ }^{b+1} 3\left(\bmod 5^{b-1}\right)$. 
As shown before, we have ${ }^{b} 3 \equiv{ }^{b+1} 3\left(\bmod \varphi\left(5^{b}\right)\right) \Rightarrow{ }^{b+1} 3 \equiv{ }^{b+2} 3\left(\bmod 5^{b}\right)$ by Euler's theorem. Since ${ }^{b} 3 \equiv{ }^{b+1} 3\left(\bmod 5^{b-1}\right) \Rightarrow{ }^{b+1} 3 \equiv{ }^{b+2} 3\left(\bmod 4 \cdot 5^{b-1}\right)$ (see $\left.[6, \mathrm{p} .66]\right)$, it follows that ${ }^{b} 3 \equiv{ }^{b+1} 3\left(\bmod 5^{b-1}\right) \Rightarrow{ }^{b+1} 3 \equiv{ }^{b+2} 3\left(\bmod 5^{b}\right)$. This confirms that the inductive step is also true.

Therefore, we have proved that

$$
{ }^{b} 3 \equiv{ }^{b+1} 3\left(\bmod 10^{\min (b+1, b-1)}\right) \Rightarrow{ }^{b} 3 \equiv{ }^{b+1} 3\left(\bmod 10^{b-1}\right) .
$$

We complete the proof of Lemma 1 showing that ${ }^{b} 3 \not{ }^{b+1} 3\left(\bmod 10^{b}\right), \forall b \in \mathbb{N}-\{0,1\}$. In order to prove that $\nexists b$ such that ${ }^{b} 3 \equiv{ }^{b+1} 3\left(\bmod 10^{b}\right)$, it is sufficient to show that ${ }^{b+1} 3 \not \equiv{ }^{b} 3\left(\bmod 5^{b}\right), \forall b$. We prove that $5^{b} \nmid{ }^{b+1} 3-{ }^{b} 3, \forall b \in \mathbb{N}-\{0,1\}$ by induction on $b$ (as usual).

Let $b=2.3^{27} \not \equiv 27(\bmod 25)$ is true, since $12 \not \equiv 2(\bmod 25)$.

We need to prove the induction step.

Let $b \geq 2$ and assume ${ }^{b+1} 3 \not{ }^{b} 3\left(\bmod 5^{b}\right)$. This is true since $5^{b-1}$ is the largest power of 5 that divides $\left({ }^{b+1} 3-{ }^{b} 3\right)$. Otherwise, $5^{b}$ would divide $\left({ }^{b+1} 3-{ }^{b} 3\right)$, which implies that " $\left({ }^{b+1} 3-{ }^{b} 3\right)$ is a multiple of $\varphi\left(5^{b+1}\right)$ ", but the statement is false since 3 is a primitive root modulo $5^{b}$ for any $b \geq 1$, and we deduce this from [5, Theorem 1]. Assuming $t=0$, [5, Theorem 1] states that if 5 is an odd prime (and it is) and if 3 is a primitive root of $5^{(b \geq 2)}$, then 3 is also a primitive root of $5^{b+1}$.

Thus, we need to check that 3 is a primitive root of $5^{2}=25$, and it is so (since $5^{2}$ has a total of 8 primitive roots: $2,3,8,12,13,17,22,23$ ).

Therefore, for $a=3$, the conguence speed of ${ }^{b} a$ is constant $(\forall b \geq 2)$.

In particular, we have shown that $V(3)=1$ (for any $b>1$ ) using the primitive root analysis, and this concludes the proof.

The primitive root argument is a key point at the bottom of many results that we will introduce in the next sections and represents a central topic discussed in [6] as well.

Property 1. $\forall a \in \mathbb{N}-\{1,2\}: a \not \equiv 0(\bmod 10), \exists b^{\prime} \in \mathbb{N}-\{0\}: b^{\prime}<a$ such that, $\forall b \geq b^{\prime}$, $V(a, b)=V(a) \in \mathbb{N}-\{0\}$ (see A317905 of the OEIS - ruling out the first term of the sequence $[9])$.

Remark 1. Referring to the aforementioned property and considering all the bases $a \in \mathbb{N}: a \not \equiv 0(\bmod 10)$, we point out that this is a peculiarity of tetration (as for the exponentiation if $a: a \equiv 0(\bmod 10))$ : from pentation (hyper-5) and beyond, $\forall a \in \mathbb{N}-\{0,1\}$, the number of stable digits will increase for any unitary increment of the hyperexponent. Perhaps we should coin the term "congruence acceleration" (rather than congruence speed), speculating that hyper-5 may be characterized by a constant congruence acceleration, for any given $a \in \mathbb{N}-\{1\}: a \not \equiv 0(\bmod 10)$.

The special feature of tetration captured by Property 1 has been widely analyzed in [6] and confirmed for specific values of $a[4,10]$, including $V(2)=1$ (for any $b \in \mathbb{N}: b \geq 3$ ) [1, p. 148]. 
Lemma 1 proves that $\exists a$ such that, $\forall b \geq 2, V(a, b)=V(a)$ is a strictly positive integer, and we are absolutely convinced that the constancy of the congruence speed (when $b \in \mathbb{N}$ is sufficiently large) is a general property [3], involving every base which is not a multiple of 10 .

Therefore, in the rest of this paper, we will assume Property 1 as a general axiom $[6,11]$.

Conjecture 1. Let $d \in \mathbb{N}_{0}$ be such that $10^{d} \mid\left({ }^{b+1} a-{ }^{b} a\right) \wedge 10^{d+1} \nmid\left({ }^{b+1} a-{ }^{b} a\right)$. Now, assume that $b \in \mathbb{N}: b \geq 3 . \forall a \in \mathbb{N}-\{1\}: a \not \equiv 0(\bmod 10)$, we have $(b-2) \cdot V(a) \leq d \leq$ $(b+1) \cdot V(a)$ (e.g., $a=2 \Rightarrow d=(b-2) \cdot(V(a)=1)$ and, $\forall k \in \mathbb{N}-\{0\}$, we have that $\left.a=2^{4 \cdot k} \Rightarrow d=(b+1) \cdot V(a)\right)$.

Remark 2. It is trivial to point out that Conjecture 1 implies that

$$
\forall a \in \mathbb{N}-\{1\}: a \not \equiv 0(\bmod 10) \wedge \forall b \in \mathbb{N}: b \geq 3,{ }^{b} a \equiv{ }^{b+1} a\left(\bmod 10^{b-2}\right) .
$$

Thus, for any $b \geq 3$, we know that the rightmost $b-2$ figures of the integer tetration ${ }^{b} a$ are stable digits: they form the same final string of ${ }^{b^{\prime}} a$, where $b^{\prime} \in \mathbb{N}: b^{\prime}>b$.

Hence, we get a conservative general upper bound $b_{u}$ for the hyperexponent $b$, applied to any base $a$ (not a multiple of 10), which assures us that all the rightmost $d$ figures of ${ }^{b^{\prime}} a$ are stable digits, for any $b^{\prime} \geq b_{u}[6]$ :

$$
b_{u}=\left\lceil\frac{d}{V(a)}\right\rceil+2
$$

(where the ceiling $\lceil q\rceil$ denotes the function that takes the rational number $q$ as input and gives as output the least integer greater than or equal to $q$ ).

Therefore, $\forall b \geq 3, V(a) \leq \frac{d}{b-2}$ (e.g., if $a=143^{625}$ and $b \geq 5$, then

$$
4=V(a) \leq \frac{0+6+6+5+\sum_{i=5}^{b} 4}{b-2}=\frac{17+(b-4) \cdot 4}{b-2}
$$

is true, while $b=4 \Rightarrow V\left(143^{625}\right) \leq \frac{17}{2}$ and $b=3 \Rightarrow V\left(143^{625}\right) \leq 12$ are trivially verified).

Furthermore, if Conjecture 1 holds, it follows that the maximum number of missed stable digits, for any given value of $a$ and for a sufficiently large $b$, is $3 \cdot V(a)$.

We can consistently define $V(1)=0$, extending the domain of $V: V(a, b)=V(a) \forall b \geq a+1$ (by Definition 1 and Property 1), if we observe that, for any $d \in \mathbb{N}, \nexists b \in \mathbb{N}-\{0,1\}$ such that

$$
{ }^{b-1} 1 \equiv{ }^{b} 1\left(\bmod 10^{d}\right) \wedge^{b-1} 1 \not \equiv{ }^{b} 1\left(\bmod 10^{d+1}\right) .
$$

Since $V(a)$ must belong to $\mathbb{N}$, it cannot be equal to $+\infty$.

In [6] the congruence speed has been introduced as the natural number which describes how many "new" stable digits appear at the beginning of the fixed figures array that is at the end of the result of the tetration, going from ${ }^{b} a$ to ${ }^{b+1} a$.

This allow us to rewrite Definition 1 as follows.

Definition 2. Let $a \in \mathbb{N}: a \not \equiv 0(\bmod 10)$, and let $b \in \mathbb{N}-\{0,1\}$ be such that $10^{d} \mid\left({ }^{b} a-{ }^{b-1} a\right) \wedge 10^{d+1} \nmid\left({ }^{b} a-{ }^{b-1} a\right)$, where $d \in \mathbb{N}$. Given $a>1$, we define $V(a) \in \mathbb{N}$ to be such that $10^{d+V(a)} \mid\left({ }^{b+1} a-{ }^{b} a\right) \wedge 10^{d+V(a)+1} \nmid\left({ }^{b+1} a-{ }^{b} a\right)$, and $V(a=1)=0$. 
Therefore, we assume that $V(1)=0$ without loss of generality, while $V(a=0)$ is not defined for the reason stated above (even if it is possible to extend the domain of tetration by considering that $\lim _{a \rightarrow 0}{ }^{b} a:={ }^{b} 0 \Rightarrow{ }^{b} 0=1$ iff $b$ is even $\wedge{ }^{b} 0=1$ otherwise [2]).

At this point, it is trivial to note that [10], $\forall b \geq a+1, V(a)=0$ iff $a \leq 1$, as shown in Table 1.

\begin{tabular}{|c|c|c|c|c|c|c|c|c|c|}
\hline$V(a)$ & 1 & 2 & 3 & 4 & 5 & 6 & 7 & 8 & 9 \\
\hline 0+ & 0 & 1 & 1 & 1 & 2 & 1 & 2 & 1 & 1 \\
\hline $10+$ & 1 & 1 & 1 & 1 & 4 & 1 & 1 & 2 & 1 \\
\hline $20+$ & 1 & 1 & 1 & 2 & 3 & 2 & 1 & 1 & 1 \\
\hline $30+$ & 1 & 2 & 1 & 1 & 2 & 1 & 1 & 1 & 1 \\
\hline $40+$ & 1 & 1 & 2 & 1 & 2 & 1 & 1 & 1 & 2 \\
\hline $50+$ & 2 & 1 & 1 & 1 & 3 & 1 & 3 & 1 & 1 \\
\hline $60+$ & 1 & 1 & 1 & 1 & 6 & 1 & 1 & 3 & 1 \\
\hline $70+$ & 1 & 1 & 1 & 2 & 2 & 2 & 1 & 1 & 1 \\
\hline $80+$ & 1 & 2 & 1 & 1 & 2 & 1 & 1 & 1 & 1 \\
\hline $90+$ & 1 & 1 & 2 & 1 & 5 & 1 & 1 & 1 & 2 \\
\hline $100+$ & 2 & 1 & 1 & 1 & 3 & 1 & 2 & 1 & 1 \\
\hline 110+ & 1 & 1 & 1 & 1 & 2 & 1 & 1 & 2 & 1 \\
\hline $120+$ & 1 & 1 & 1 & 3 & 2 & 3 & 1 & 1 & 1 \\
\hline 130+ & 1 & 2 & 1 & 1 & 3 & 1 & 1 & 1 & 1 \\
\hline 140+ & 1 & 1 & 2 & 1 & 4 & 1 & 1 & 1 & 2 \\
\hline $150+$ & 2 & 1 & 1 & 1 & 2 & 1 & 2 & 1 & 1 \\
\hline $160+$ & 1 & 1 & 1 & 1 & 2 & 1 & 1 & 2 & 1 \\
\hline 170+ & 1 & 1 & 1 & 2 & 4 & 2 & 1 & 1 & 1 \\
\hline $180+$ & 1 & 4 & 1 & 1 & 3 & 1 & 1 & 1 & 1 \\
\hline 190+ & 1 & 1 & 3 & 1 & 2 & 1 & 1 & 1 & 2 \\
\hline $200+$ & 2 & 1 & 1 & 1 & 2 & 1 & 2 & 1 & 1 \\
\hline $210+$ & 1 & 1 & 1 & 1 & 3 & 1 & 1 & 2 & 1 \\
\hline $220+$ & 1 & 1 & 1 & 2 & 5 & 2 & 1 & 1 & 1 \\
\hline 230+ & 1 & 2 & 1 & 1 & 2 & 1 & 1 & 1 & 1 \\
\hline $240+$ & 1 & 1 & 2 & 1 & 2 & 1 & 1 & 1 & 3 \\
\hline $250+$ & 2 & 1 & 1 & 1 & 8 & 1 & 2 & 1 & 1 \\
\hline $260+$ & 1 & 1 & 1 & 1 & 3 & 1 & 1 & 2 & 1 \\
\hline $270+$ & 1 & 1 & 1 & 2 & 2 & 2 & 1 & 1 & 1 \\
\hline $280+$ & 1 & 2 & 1 & 1 & 2 & 1 & 1 & 1 & 1 \\
\hline $290+$ & 1 & 1 & 2 & 1 & 3 & 1 & 1 & 1 & 2 \\
\hline 300+ & 2 & 1 & 1 & 1 & 4 & 1 & 2 & 1 & 1 \\
\hline 310+ & 1 & 1 & 1 & 1 & 2 & 1 & 1 & 3 & 1 \\
\hline $320+$ & 1 & 1 & 1 & 2 & 2 & 2 & 1 & 1 & 1 \\
\hline 330+ & 1 & 2 & 1 & 1 & 4 & 1 & 1 & 1 & 1 \\
\hline 340+ & 1 & 1 & 2 & 1 & 3 & 1 & 1 & 1 & 2 \\
\hline $350+$ & 2 & 1 & 1 & 1 & 2 & 1 & 2 & 1 & 1 \\
\hline $360+$ & 1 & 1 & 1 & 1 & 2 & 1 & 1 & 2 & 1 \\
\hline 370+ & 1 & 1 & 1 & 3 & 3 & 3 & 1 & 1 & 1 \\
\hline 380+ & 1 & 2 & 1 & 1 & 7 & 1 & 1 & 1 & 1 \\
\hline 390+ & 1 & 1 & 2 & 1 & 2 & 1 & 1 & 1 & 2 \\
\hline
\end{tabular}




\begin{tabular}{|c|c|c|c|c|c|c|c|c|c|}
\hline$V(a)$ & 1 & 2 & 3 & 4 & 5 & 6 & 7 & 8 & 9 \\
\hline $400+$ & 2 & 1 & 1 & 1 & 2 & 1 & 2 & 1 & 1 \\
\hline $410+$ & 1 & 1 & 1 & 1 & 5 & 1 & 1 & 2 & 1 \\
\hline $420+$ & 1 & 1 & 1 & 2 & 3 & 2 & 1 & 1 & 1 \\
\hline $430+$ & 1 & 3 & 1 & 1 & 2 & 1 & 1 & 1 & 1 \\
\hline $440+$ & 1 & 1 & 2 & 1 & 2 & 1 & 1 & 1 & 2 \\
\hline $450+$ & 2 & 1 & 1 & 1 & 3 & 1 & 2 & 1 & 1 \\
\hline $460+$ & 1 & 1 & 1 & 1 & 4 & 1 & 1 & 2 & 1 \\
\hline 470+ & 1 & 1 & 1 & 2 & 2 & 2 & 1 & 1 & 1 \\
\hline $480+$ & 1 & 2 & 1 & 1 & 2 & 1 & 1 & 1 & 1 \\
\hline $490+$ & 1 & 1 & 2 & 1 & 4 & 1 & 1 & 1 & 2 \\
\hline $500+$ & 2 & 1 & 1 & 1 & 3 & 1 & 2 & 1 & 1 \\
\hline $510+$ & 1 & 1 & 1 & 1 & 2 & 1 & 1 & 2 & 1 \\
\hline $520+$ & 1 & 1 & 1 & 2 & 2 & 2 & 1 & 1 & 1 \\
\hline 530+ & 1 & 2 & 1 & 1 & 3 & 1 & 1 & 1 & 1 \\
\hline $540+$ & 1 & 1 & 2 & 1 & 5 & 1 & 1 & 1 & 2 \\
\hline $550+$ & 2 & 1 & 1 & 1 & 2 & 1 & 2 & 1 & 1 \\
\hline $560+$ & 1 & 1 & 1 & 1 & 2 & 1 & 1 & 3 & 1 \\
\hline 570+ & 1 & 1 & 1 & 2 & 6 & 2 & 1 & 1 & 1 \\
\hline $580+$ & 1 & 2 & 1 & 1 & 3 & 1 & 1 & 1 & 1 \\
\hline 590+ & 1 & 1 & 2 & 1 & 2 & 1 & 1 & 1 & 2 \\
\hline $600+$ & 2 & 1 & 1 & 1 & 2 & 1 & 2 & 1 & 1 \\
\hline $610+$ & 1 & 1 & 1 & 1 & 3 & 1 & 1 & 2 & 1 \\
\hline $620+$ & 1 & 1 & 1 & 4 & 4 & 4 & 1 & 1 & 1 \\
\hline 630+ & 1 & 2 & 1 & 1 & 2 & 1 & 1 & 1 & 1 \\
\hline $640+$ & 1 & 1 & 2 & 1 & 2 & 1 & 1 & 1 & 2 \\
\hline $650+$ & 2 & 1 & 1 & 1 & 4 & 1 & 2 & 1 & 1 \\
\hline $660+$ & 1 & 1 & 1 & 1 & 3 & 1 & 1 & 2 & 1 \\
\hline $670+$ & 1 & 1 & 1 & 2 & 2 & 2 & 1 & 1 & 1 \\
\hline $680+$ & 1 & 3 & 1 & 1 & 2 & 1 & 1 & 1 & 1 \\
\hline 690+ & 1 & 1 & 2 & 1 & 3 & 1 & 1 & 1 & 2 \\
\hline 700+ & 2 & 1 & 1 & 1 & 6 & 1 & 2 & 1 & 1 \\
\hline $710+$ & 1 & 1 & 1 & 1 & 2 & 1 & 1 & 2 & 1 \\
\hline $720+$ & 1 & 1 & 1 & 2 & 2 & 2 & 1 & 1 & 1 \\
\hline 730+ & 1 & 2 & 1 & 1 & 5 & 1 & 1 & 1 & 1 \\
\hline $740+$ & 1 & 1 & 2 & 1 & 3 & 1 & 1 & 1 & 2 \\
\hline $750+$ & 3 & 1 & 1 & 1 & 2 & 1 & 2 & 1 & 1 \\
\hline $760+$ & 1 & 1 & 1 & 1 & 2 & 1 & 1 & 2 & 1 \\
\hline $770+$ & 1 & 1 & 1 & 2 & 3 & 2 & 1 & 1 & 1 \\
\hline $780+$ & 1 & 2 & 1 & 1 & 4 & 1 & 1 & 1 & 1 \\
\hline 790+ & 1 & 1 & 2 & 1 & 2 & 1 & 1 & 1 & 2 \\
\hline $800+$ & 2 & 1 & 1 & 1 & 2 & 1 & 3 & 1 & 1 \\
\hline $810+$ & 1 & 1 & 1 & 1 & 4 & 1 & 1 & 3 & 1 \\
\hline $820+$ & 1 & 1 & 1 & 2 & 3 & 2 & 1 & 1 & 1 \\
\hline $830+$ & 1 & 2 & 1 & 1 & 2 & 1 & 1 & 1 & 1 \\
\hline $840+$ & 1 & 1 & 2 & 1 & 2 & 1 & 1 & 1 & 2 \\
\hline $850+$ & 2 & 1 & 1 & 1 & 3 & 1 & 2 & 1 & 1 \\
\hline $860+$ & 1 & 1 & 1 & 1 & 5 & 1 & 1 & 2 & 1 \\
\hline $870+$ & 1 & 1 & 1 & 3 & 2 & 3 & 1 & 1 & 1 \\
\hline $880+$ & 1 & 2 & 1 & 1 & 2 & 1 & 1 & 1 & 1 \\
\hline $890+$ & 1 & 1 & 2 & 1 & 7 & 1 & 1 & 1 & 2 \\
\hline
\end{tabular}




\begin{tabular}{|l|l|l|l|l|l|l|l|l|l|}
\hline $\boldsymbol{V}(\boldsymbol{a})$ & $\mathbf{1}$ & $\mathbf{2}$ & $\mathbf{3}$ & $\mathbf{4}$ & $\mathbf{5}$ & $\mathbf{6}$ & $\mathbf{7}$ & $\mathbf{8}$ & $\mathbf{9}$ \\
\hline $\mathbf{9 0 0 +}$ & 2 & 1 & 1 & 1 & 3 & 1 & 2 & 1 & 1 \\
\hline $\mathbf{9 1 0 +}$ & 1 & 1 & 1 & 1 & 2 & 1 & 1 & 2 & 1 \\
\hline $\mathbf{9 2 0 +}$ & 1 & 1 & 1 & 2 & 2 & 2 & 1 & 1 & 1 \\
\hline $\mathbf{9 3 0 +}$ & 1 & 3 & 1 & 1 & 3 & 1 & 1 & 1 & 1 \\
\hline $\mathbf{9 4 0 +}$ & 1 & 1 & 3 & 1 & 4 & 1 & 1 & 1 & 2 \\
\hline $\mathbf{9 5 0 +}$ & 2 & 1 & 1 & 1 & 2 & 1 & 2 & 1 & 1 \\
\hline $\mathbf{9 6 0 +}$ & 1 & 1 & 1 & 1 & 2 & 1 & 1 & 2 & 1 \\
\hline $\mathbf{9 7 0 +}$ & 1 & 1 & 1 & 2 & 4 & 2 & 1 & 1 & 1 \\
\hline $\mathbf{9 8 0 +}$ & 1 & 2 & 1 & 1 & 3 & 1 & 1 & 1 & 1 \\
\hline $\mathbf{9 9 0 +}$ & 1 & 1 & 2 & 1 & 2 & 1 & 1 & 1 & 3 \\
\hline $\mathbf{1 0 0 0 +}$ & 3 & 1 & 1 & 1 & 2 & 1 & 2 & 1 & 1 \\
\hline $\mathbf{1 0 1 0 +}$ & 1 & 1 & 1 & 1 & 3 & 1 & 1 & 2 & 1 \\
\hline $\mathbf{1 0 2 0 +}$ & 1 & 1 & 1 & 2 & 10 & 2 & 1 & 1 & 1 \\
\hline $\mathbf{1 0 3 0 +}$ & 1 & 2 & 1 & 1 & 2 & 1 & 1 & 1 & 1 \\
\hline $\mathbf{1 0 4 0 +}$ & 1 & 1 & 2 & 1 & 2 & 1 & 1 & 1 & 2 \\
\hline $\mathbf{1 0 5 0 +}$ & 2 & 1 & 1 & 1 & 5 & 1 & 3 & 1 & 1 \\
\hline $\mathbf{1 0 6 0 +}$ & 1 & 1 & 1 & 1 & 3 & 1 & 1 & 6 & 1 \\
\hline $\mathbf{1 0 7 0 +}$ & 1 & 1 & 1 & 2 & 2 & 2 & 1 & 1 & 1 \\
\hline $\mathbf{1 0 8 0}+$ & 1 & 2 & 1 & 1 & 2 & 1 & 1 & 1 & 1 \\
\hline $\mathbf{1 0 9 0 +}$ & 1 & 1 & 2 & 1 & 3 & 1 & 1 & 1 & 2 \\
\hline $\mathbf{1 1 0 0 +}$ & 2 & 1 & 1 & 1 & 4 & 1 & 2 & 1 & 1 \\
\hline
\end{tabular}

Table 1: $V(a)$ for $a \leq 1109$. Given $a>1$ such that $a \not \equiv 0(\bmod 10)$, if $V(a) \leq 2$, then $V(a)=V(a+k \cdot 1000)$ for any $k \in \mathbb{N}_{0}$ (by Hypothesis 2 ).

Hypothesis 1. $\forall a \in \mathbb{N}-\{1,2\}: a \not \equiv 0(\bmod 10), \exists b^{\prime} \in \mathbb{N}-\{0\}: b^{\prime}<a$ such that, $\forall b \geq b^{\prime}$, $V(a)=1 \Leftrightarrow a(\bmod 25) \in \mathbb{C}^{C}=\{2,3,4,6,8,9,11,12,13,14,16,17,19,21,22,23\}$
$V(a) \geq 2 \Leftrightarrow a(\bmod 25) \in \mathbb{C}=\{0,1,5,7,10,15,18,20,24\}$.

Remark 3. It is important to notice that, if $a(\bmod 25) \in \mathbb{C}^{C}$ (or equivalently if $V(a)=1$ ), then $V\left(a^{m}\right) \geq 2 \forall m=5 \cdot n$ (where $n \in \mathbb{N}-\{0\}$ ), and $V\left(a^{m}\right)=1$ otherwise (for any $m$ such that $m(\bmod 10) \equiv\{1,2,3,4,6,7,8,9\})$.

On the contrary, for any base $a($ not a multiple of 10$)$ such that $a(\bmod 25) \in \mathbb{C}, V\left(a^{n}\right) \geq 2$ (since also $\left.a^{n}(\bmod 25) \in \mathbb{C} \forall n \in \mathbb{N}-\{0\}\right)$. We point out that

$$
V(a) \geq 2 \Rightarrow a^{m+1}(\bmod 25) \equiv a(\bmod 25), \forall m=4 \cdot n \text {. }
$$

Proposition 1. Let $a \in \mathbb{N}$ be such that $a \not \equiv 0(\bmod 10) . \forall k \in \mathbb{N}, a^{20 \cdot k+1} \equiv a(\bmod 25)$.

Proof. $\forall n \in \mathbb{N}-\{0\}$, we have $\lambda(n) \leq \varphi(n)$. Thus, $\lambda(25)=\varphi(25)=20$.

Let $a$ be such that

$$
\operatorname{gcd}(a, 25)=1 \Leftrightarrow \operatorname{gcd}(a, 5)=1, a^{\lambda(25)} \equiv 1(\bmod 25) \Rightarrow a^{20+1} \equiv a(\bmod 25) .
$$

Hence $a^{20 \cdot k+1} \equiv a(\bmod 25)$.

Let $a$ be such that

$$
a \equiv 5(\bmod 10), \forall m \in \mathbb{N}-\{0,1\}, a^{m} \equiv 0(\bmod 25) \Rightarrow a^{m} \equiv a^{m+1}(\bmod 25) .
$$

Therefore, $a^{2}(\bmod 25) \equiv a^{3}(\bmod 25) \equiv \cdots \equiv a^{20+1}(\bmod 25) \equiv \cdots \equiv a^{20 \cdot k+1}(\bmod 25)$. 
Hypothesis 2. $\forall a \in \mathbb{N}-\{1,2\}: a \not \equiv 0(\bmod 10), \exists b^{\prime} \in \mathbb{N}-\{0\}: b^{\prime}<a$ such that, $\forall b \geq b^{\prime}$,

$$
\left\{\begin{array}{c}
V(a)=1 \Leftrightarrow a(\bmod 25) \in \mathbb{C}^{C}=\{2,3,4,6,8,9,11,12,13,14,16,17,19,21,22,23\} ; \\
V(a)=2 \Leftrightarrow a(\bmod 40) \in\{5,35\} \vee \\
\left(a(\bmod 25) \in\{1,7,18,24\} \wedge a(\bmod 1000) \notin \mathbb{Q}^{C}\right) ; \\
V(a) \geq 3 \Leftrightarrow a(\bmod 40) \in\{15,25\} \vee a(\bmod 1000) \in \mathbb{Q}^{C} \\
\text { where } \mathbb{Q}^{C}=\left\{\begin{array}{c}
1,57,68,124,126,182,193,249,318,374,376,432,568, \\
624,626,682,751,807,818,874,876,932,943,999
\end{array}\right\} .
\end{array}\right.
$$

Remark 4. We theorize that $V(a)=v$ originates a cycle for any $v \in \mathbb{N}-\{0\}$, where the fundamental period $P(v)$ is such that $P(1)=25, P(2)=10^{3}, P(3)=10^{4}$, etc.

Additionally, we presume that, $\forall b \in \mathbb{N}: b \geq a, V(a \equiv 4(\bmod 10))=V(a \equiv 6(\bmod 10))$ as a consequence of the size of the set $\mathbb{C}^{C} \cup \mathbb{C}$.

Proposition 2. Let $\operatorname{len}\left(a_{i}\right) \in \mathbb{N}: 10^{\operatorname{len}\left(a_{i}\right)-1}<a_{i}<10^{\operatorname{len}\left(a_{i}\right)}$ denote the number of digits of the $i$-th term of any integer sequence $a_{n}:=s_{1}, s_{2} s_{1}, \ldots, s_{i-1} \ldots s_{2} s_{1}, s_{i} s_{i-1} \ldots s_{2} s_{1}, \ldots$ constructed through the juxtaposition of its elements (i.e., $\forall n \in \mathbb{N}-\{0,1\} a_{n}:=s_{n} a_{n-1}$, where $s_{n} \in \mathbb{N}_{0}$ is arbitrary). Given $s_{1} \not \equiv 0(\bmod 10), \forall i \in \mathbb{N}$ such that $\operatorname{len}\left(a_{i}\right) \geq 2$, we have ${ }^{a_{i}} a_{i} \equiv{ }^{a_{i+1}} a_{i+1}\left(\bmod 10^{\operatorname{len}\left(a_{i}\right)}\right)$.

Proof. This particularity involving iterated exponentiation has been discussed in [6, p. 60], and two examples of the aforementioned property are given by the sequences A317824 and A317903 of the OEIS [7, 8].

In [10] the authors proved that, $\forall a \in \mathbb{N}: a \not \equiv 0(\bmod 10) \wedge \forall n \in \mathbb{N}: n \geq 2$, there exists a unique (potentially unlimited) sequence of stable digits, that they indicated as $x_{n}(a):=x_{n-1} \ldots x_{2} x_{1} x_{0}$, such that ${ }^{b} a \equiv x_{n}(a)\left(\bmod 10^{n}\right)$ for a sufficiently large value of $b$.

Now, let $n:=\operatorname{len}\left(a_{i}\right)$ and assume $n \geq 2$. Since $b=a$, the conditions stated in [4, 10] are always satisfied. This implies that, $\forall \operatorname{len}\left(a_{i}\right) \geq 2$, if the congruence speed of any $a>9$ (not a multiple of 10) is constant, then it is always greater or equal than 1 (see [10], Theorem 3, proof of Case I and Case II considering radix-10).

Therefore (by Property 1), we conclude that the property captured by Proposition 2 definitely holds for any $a \not \equiv 0(\bmod 10)$.

Conjecture 2. Let $|x|$ denote the absolute value of $x$ and let $d \in \mathbb{N}-\{0\}$ be such that ${ }^{b} a \equiv{ }^{b+1} a\left(\bmod 10^{d}\right) \wedge{ }^{b} a \not \equiv{ }^{b+1} a\left(\bmod 10^{d+1}\right) . \quad \forall a \in \mathbb{N}: a \equiv\{1,3,5,7,9\}(\bmod 10)$ and $\forall b \in \mathbb{N}-\{1\}: b \geq a$, we have

$$
\left|\frac{{ }^{b+1} a\left(\bmod 10^{d+1}\right)-{ }^{b} a\left(\bmod 10^{d+1}\right)}{10^{d}}\right|=\left|\frac{b+2 a\left(\bmod 10^{d+1+V(a)}\right)-{ }^{b+1} a\left(\bmod 10^{d+1+V(a)}\right)}{10^{d+V(a)}}\right| .
$$

Otherwise, $\forall a \in \mathbb{N}: a \equiv\{2,4,6,8\}(\bmod 10)$ and $\forall b \in \mathbb{N}-\{2\}: b \geq a$, we have

$$
\left|\frac{b+1}{b\left(\bmod 10^{d+1}\right)-{ }^{b} a\left(\bmod 10^{d+1}\right)}\right|=\left|\frac{{ }^{b+3} a\left(\bmod 10^{d+1+2 \cdot V(a)}\right)-{ }^{b+2} a\left(\bmod 10^{d+1+2 \cdot V(a)}\right)}{10^{d}}\right| .
$$

Remark 5. In [6] it has already been shown the structure and the size of the cycles involving up to 4 figures on the left of the leftmost stable digit of ${ }^{b} a$, for any given $b \geq a$ (see [6, pp. 49-59]). 


\section{Main result: finding $V(a)$ for any $a: a \equiv 5(\bmod 10)$}

In the present section, we consider only bases of the tetration ${ }^{b} a$ such that their ending digit is 5 . Thus let $a: a \equiv 5(\bmod 10)$.

As shown in 2011 [6, pp. 22-23], given

$$
w \in \mathbb{N}: w=2^{m} \cdot \prod_{i \geq 2} p_{i} c_{i}, V\left(a^{w}\right)=V\left(a^{\left(2^{m}\right)}\right)=q+m,
$$

where $q$ depends only on $a$, while $m$ corresponds to the highest power of 2 that appears in the factorization of the exponent of $a$.

If $a^{*}:=5^{\left(2^{m}\right)}$, then $V\left(a^{*}\right)=V(a=5)+m=2+m\left(\forall m \in \mathbb{N}_{0}\right)$. So, if we wish to construct a base $a \equiv 5(\bmod 10)$ such that $V(a)=1729$, we can simply take $a^{*}=5^{\left(2^{1729-2}\right)}=5^{\left(2^{1727}\right)}$.

In general, let $v$ be any integer greater or equal to 2 . We can choose any $v$ and take $a=5^{\left(2^{v-2}\right)}$ in order to satisfy $v=V(a: a \equiv 5(\bmod 10))$, but the next natural question would be: "Is $a=5^{\left(2^{m}\right)}$ the smallest base such that, $\forall v \in \mathbb{N}-\{0-1\}, v=V(a: a \equiv 5(\bmod 10))$ ?".

The negative answer trivially follows from Table 1 .

Moreover, given $a \equiv 5(\bmod 10)$ as usual, we can see that

$$
\begin{aligned}
& V(a)=2 \Rightarrow a:\left\{\begin{array}{c}
a \equiv 5(\bmod 40) \\
a \equiv 35(\bmod 40)
\end{array}, \quad V(a)=3 \Rightarrow a:\left\{\begin{array}{l}
a \equiv 25(\bmod 80) \\
a \equiv 55(\bmod 80)
\end{array},\right.\right. \\
& V(a)=4 \Rightarrow a:\left\{\begin{array}{c}
a \equiv 15(\bmod 160) \\
a \equiv 145(\bmod 160)
\end{array}, \quad V(a)=5 \Rightarrow a:\left\{\begin{array}{c}
a \equiv 95(\bmod 320) \\
a \equiv 225(\bmod 320)
\end{array},\right.\right. \\
& V(a)=6 \Rightarrow a:\left\{\begin{array}{c}
a \equiv 65(\bmod 640) \\
a \equiv 575(\bmod 640)
\end{array}, \quad V(a)=7 \Rightarrow a:\left\{\begin{array}{l}
a \equiv 385(\bmod 1280) \\
a \equiv 895(\bmod 1280)
\end{array},\right.\right. \\
& V(a)=8 \Rightarrow a:\left\{\begin{array}{c}
a \equiv 255(\bmod 2560) \\
a \equiv 2305(\bmod 2560)
\end{array}, \quad V(a)=9 \Rightarrow a:\left\{\begin{array}{l}
a \equiv 1535(\bmod 5120) \\
a \equiv 3585(\bmod 5120)
\end{array},\right.\right. \\
& V(a)=10 \Rightarrow a:\left\{\begin{array}{l}
a \equiv 1025(\bmod 10240) \\
a \equiv 9215(\bmod 10240)
\end{array} \quad\right. \text { and so on. }
\end{aligned}
$$

Thus, for every $m \geq 2$, we can easily define short bases $\tilde{a}<5^{\left(2^{m}\right)}$ such that $V(\tilde{a})=v$. Furthermore, $\tilde{a}:=(a: V(a)=v)+10 \cdot 2^{v-1} \Rightarrow V(\tilde{a}) \geq v+1$, as shown in Figures 1 and 2 . 


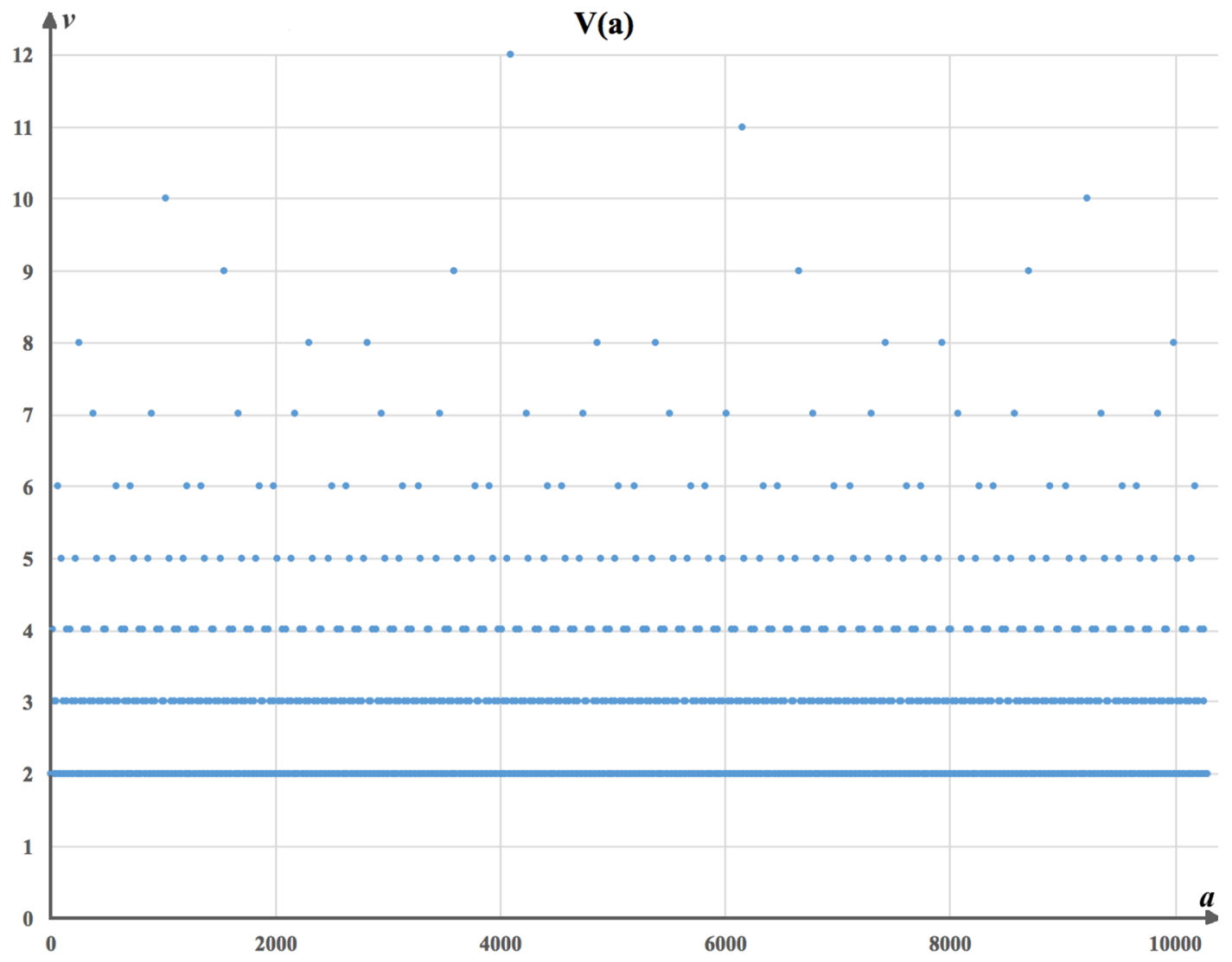

Figure 1. $V(a)$ for $5 \leq a \leq 10285$, where $a: a \equiv 5(\bmod 10)$.

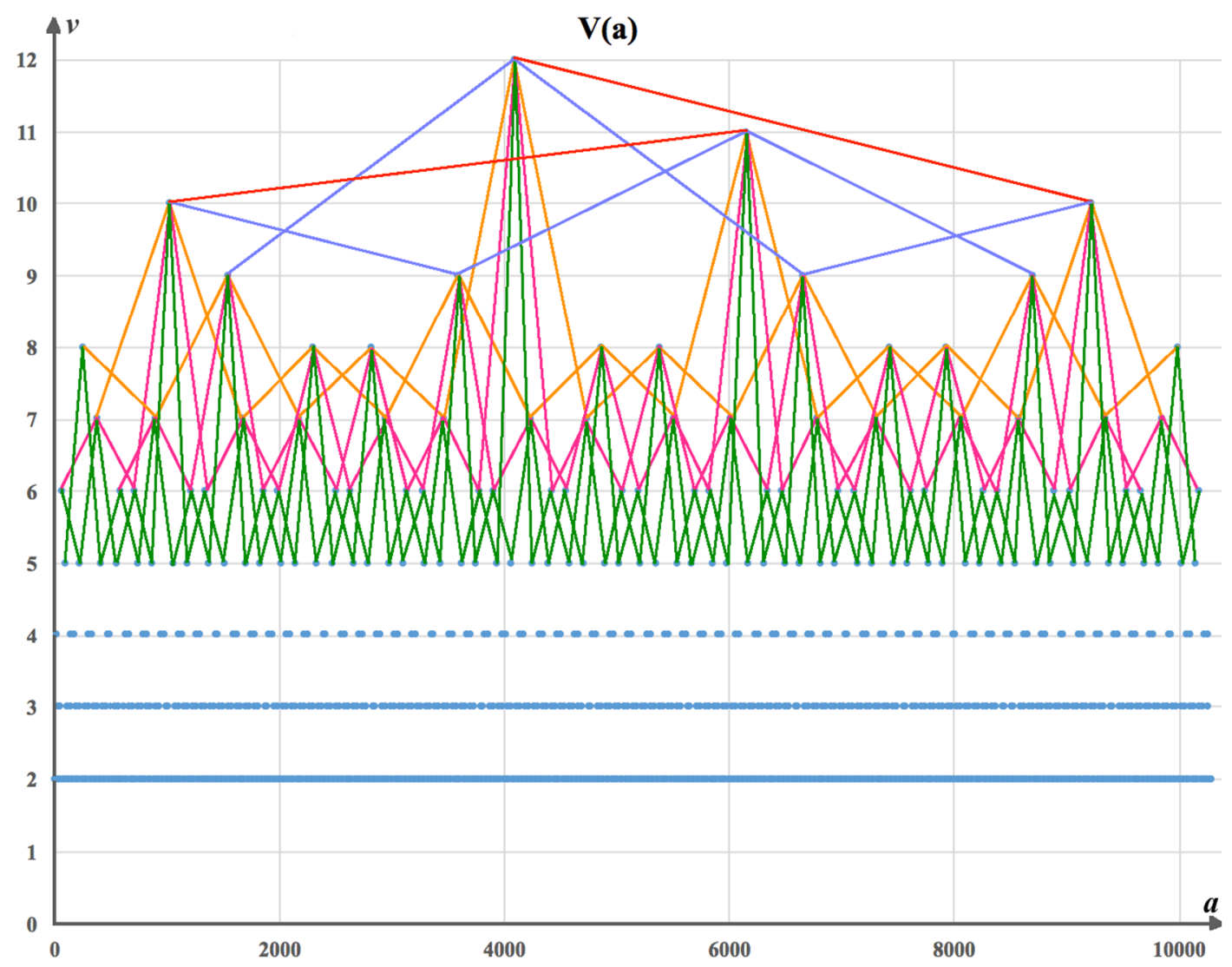

Figure 2. Period of $a: V(a)=v \geq 5$, where $a \leq 10285$ is such that $a \equiv 5(\bmod 10)$. 
We can easily find one half of all the (infinite) bases such that $v=V(a: a \equiv 5(\bmod 10))$. If $V\left(5^{\left(2^{m}\right)}\right)=V(5)+m$ (see [6, pp. 16, 22-23]), it follows that $V\left(5^{\left(2^{m}\right)}+k \cdot 10 \cdot 2^{m+2}\right)=$ $2+m$. This means that also

$$
V\left(5 \cdot 2^{m+2}+1+\frac{2^{\left(4 \cdot\left[\frac{m-2}{4} \mid+2\right)\right.}-2^{\left(4 \cdot\left|\frac{m}{4}\right|+6\right)}}{3}+k \cdot 10 \cdot 2^{m+2}\right)
$$

is equal to $2+m$.

Now, $\forall m \in \mathbb{N}_{0}$, it is clear that the other half of the values have to be such that, $\forall k \in \mathbb{N}_{0}$,

$$
V(a)=2+m \Leftarrow a=5 \cdot 2^{m+2}-1-\frac{2^{\left(4 \cdot\left[\frac{m-2}{4}\right\rfloor+2\right)}-2\left(4 \cdot\left[\frac{m}{4}\right\rfloor+6\right)}{3}+k \cdot 5 \cdot 2^{m+3} .
$$

Thus, $\forall a: a \equiv 5(\bmod 10)$, if $V(a)=2+m$, then

$$
a:\left\{\begin{array}{l}
a=5 \cdot 2^{m+2}-2^{m+4} \cdot \sin \left(\frac{\pi \cdot(m+1)}{2}\right)+2^{m+3} \cdot \cos \left(\frac{\pi \cdot(m+1)}{2}\right)+1+k \cdot 5 \cdot 2^{m+3} \\
a=5 \cdot 2^{m+2}+2^{m+4} \cdot \sin \left(\frac{\pi \cdot(m+1)}{2}\right)-2^{m+3} \cdot \cos \left(\frac{\pi \cdot(m+1)}{2}\right)-1+k \cdot 5 \cdot 2^{m+3}
\end{array} .\right.
$$

Hence, $\forall k \in \mathbb{N}_{0}$,

$$
V(a)=2+m \Rightarrow a:\left\{\begin{array}{l}
a=2^{m+3} \cdot\left(\cos \left(\frac{\pi \cdot(m+1)}{2}\right)-2 \cdot \sin \left(\frac{\pi \cdot(m+1)}{2}\right)+5 \cdot\left(k+\frac{1}{2}\right)\right)+1 \\
a=2^{m+3} \cdot\left(2 \cdot \sin \left(\frac{\pi \cdot(m+1)}{2}\right)-\cos \left(\frac{\pi \cdot(m+1)}{2}\right)+5 \cdot\left(k+\frac{1}{2}\right)\right)-1
\end{array} .\right.
$$

Theorem 1. Let $a: a \equiv 5(\bmod 10)$ be the base of the tetration ${ }^{b} a$. Let $b \in \mathbb{N}: b \geq 3$. $\forall k \in \mathbb{N}_{0}, V(a)=2+m \Leftrightarrow a=\left(x_{m} \vee y_{m}\right)+k \cdot 10 \cdot 2^{m+2}$, where $\left(\forall m \in \mathbb{N}_{0}\right)$ $x_{m}:=2^{m+2} \cdot\left(2 \cdot \cos \left(\frac{\pi \cdot(m+1)}{2}\right)-4 \cdot \sin \left(\frac{\pi \cdot(m+1)}{2}\right)+5\right)+1=(5,25,145,225,65,385, \ldots)$ and $y_{m}:=2^{m+2} \cdot\left(4 \cdot \sin \left(\frac{\pi \cdot(m+1)}{2}\right)-2 \cdot \cos \left(\frac{\pi \cdot(m+1)}{2}\right)+5\right)-1=$ $(35,55,15,95,575,895, \ldots)$. Furthermore, for any $a, b$, and $m$ as above, we have

$$
{ }^{b} a \equiv{ }^{b+1} a\left(\bmod 10^{d}\right) \wedge{ }^{b} a \not{ }^{b+1} a\left(\bmod 10^{d+1}\right) \Leftrightarrow d=(2+m) \cdot(b+1) .
$$

Proof. Let $a: a \equiv 5(\bmod 10)$ be given. If $b \geq a$, then $v=V(a)$ is independent from $b$ (by Property 1).

In [6, pp. 16, 19-20] it has been shown that the constraint $b \geq a$ is a sufficient but not a necessary condition. In particular, for any given $a: a \equiv 5(\bmod 10)$, if $b \geq 3$, then $V(a, b)=V(a)$.

Now, if ${ }^{b} a \equiv{ }^{b+1} a\left(\bmod 10^{d}\right) \wedge{ }^{b} a \not{ }^{b+1} a\left(\bmod 10^{d+1}\right) \Rightarrow d=(2+m) \cdot(b+1)$, then $10^{b \cdot(2+m)+m+2} \mid\left({ }^{b+1} a-{ }^{b} a\right) \wedge 10^{b \cdot(2+m)+m+3} \nmid\left({ }^{b+1} a-{ }^{b} a\right) \Rightarrow d=(2+m) \cdot(b+1)$.

Hence,

$$
10^{v \cdot(b+1)} \mid\left({ }^{b+1} a-{ }^{b} a\right) \wedge 10^{v \cdot(b+1)+1} \nmid\left({ }^{b+1} a-{ }^{b} a\right) \Rightarrow d=v \cdot(b+1) .
$$

Therefore, given $b \geq 3$, we have $V(a, 1))+V(a, 2))=3 \cdot V(a, b))$, for any $a$ such that $a \equiv 5(\bmod 10)$. 
Thus, $d=3 \cdot v+(b-2) \cdot v$, where $d$ represents the maximum number of stable digits originated by ${ }^{b} a$, for any given $b \geq 3$ (see Definitions 1 and 2). This proves the last statement of Theorem 1.

In order to prove that the main statement is also true, we may observe that

$$
5^{\left(2^{m}\right)}\left(\bmod 5 \cdot 2^{m+3}\right) \equiv 5 \cdot 2^{m+2}-2^{m+4} \cdot \sin \left(\frac{\pi \cdot(m+1)}{2}\right)+2^{m+3} \cdot \cos \left(\frac{\pi \cdot(m+1)}{2}\right)+1 .
$$

Hence, $\forall m, k \in \mathbb{N}_{0}, V\left(a^{*}:=5^{\left(2^{m}\right)}+k \cdot 10 \cdot 2^{m+2}\right)=2+m$.

Thus, $a^{*}=x_{m}+k \cdot 10 \cdot 2^{m+2} \Rightarrow V\left(a^{*}\right)=2+m=V(a)$.

Let $a^{* *}$ be the other half of the bases. $a^{* *}$ is such that, $\forall m \in \mathbb{N}_{0}, V(a)=2+m \Rightarrow$ $a: a=\left(a^{*} \vee a^{* *}\right)$, and $a^{* *}$ has the same fundamental period of $a^{*}$, so $V\left(a^{* *}+5 \cdot(-2)^{m+2}\right)=V\left(a^{* *}\right)+1$ if and only if $V\left(a^{*}\right)+1=V\left(a^{*}+5 \cdot(-2)^{m+2}\right)$.

In fact, $\forall m \in \mathbb{N}_{0}$, we have $a^{*}\left(\bmod 5 \cdot 2^{m+3}\right)+a^{* *}\left(\bmod 5 \cdot 2^{m+3}\right)=5 \cdot 2^{m+3}$.

Moreover, (given $a: a \equiv 5(\bmod 10)$ as usual), we can take one solution $a_{(m=0)} \equiv 35(\bmod 40)$ of $V(a \equiv 35(\bmod 40))=2 \quad($ since we need a base such that $V(a \not \equiv 5(\bmod 20))=2)$, add it to $5 \cdot(-2)^{0+2}$, and compute the residue modulo $5 \cdot 2^{4}$. So, let $a_{0}=35$. In general, $\forall k$, we have $a_{m+1} \equiv\left(a_{m}+5 \cdot(-2)^{m+2}\right)\left(\bmod 5 \cdot 2^{m+4}\right) \Rightarrow V\left(a_{m+1}\right)=$ $V\left(a_{m}+k \cdot 10 \cdot 2^{m+2}\right)+1=m+3$.

Thus,

$$
V\left(y_{m}:=2^{m+2} \cdot\left(4 \cdot \sin \left(\frac{\pi \cdot(m+1)}{2}\right)-2 \cdot \cos \left(\frac{\pi \cdot(m+1)}{2}\right)+5\right)-1\right)=2+m=V\left(x_{m}\right) .
$$

If $a: a \equiv 5(\bmod 10)$ does not belong to $y_{m}$, then it must belong to $x_{m}$ (and vice versa). Therefore, we have also shown that

$$
V(a)=2+m \Rightarrow a=\left(x_{m}+k \cdot 10 \cdot 2^{m+2} \vee y_{m}+k \cdot 10 \cdot 2^{m+2}\right),
$$

and this completes the proof of Theorem 1.

Corollary 1. Let $\delta$ represent the Kronecker delta, the function of two variables defined by $\delta_{i, j}:=\left\{\begin{array}{l}0 \text { if } i \neq j \\ 1 \text { if } i=j\end{array}\right.$ Let $n \in \mathbb{N}_{0}$. Let $a_{n}:=5+n \cdot 10$ be the base of the tetration ${ }^{b} a_{n}$, where $b \in \mathbb{N}: b \geq 3 . \forall v \in \mathbb{N}-\{0,1\}$, we have

$$
\lim _{N \rightarrow+\infty}\left(\frac{\sum_{n=0}^{N} \delta_{V\left(a_{n}\right), v}}{\sum_{n=0}^{N} \delta_{V\left(a_{n}\right), v+1}}\right)=2 .
$$

Proof. Assume $a$ be such that $a \equiv 5(\bmod 10)$. Then, $\forall b \in \mathbb{N}: b \geq 3, V(a, b)=V(a)$ (this follows from Property 1 and Theorem 1$)$.

Let $k, n \in \mathbb{N}_{0}$. Assume $m \in \mathbb{N}_{0}$ is given. By Theorem 1 , if $a_{n}:=5+n \cdot 10$ and $b \geq 3$, then

$$
V\left(a_{n}\right)=m+2 \Leftrightarrow a_{n}=2^{m+2} \cdot\left(4 \cdot \sin \left(\frac{\pi \cdot(m+1)}{2}\right)-2 \cdot \cos \left(\frac{\pi \cdot(m+1)}{2}\right)+5\right)-1+k \cdot 5 \cdot 2^{m+3}
$$

or

$$
a_{n}=2^{m+2} \cdot\left(-4 \cdot \sin \left(\frac{\pi \cdot(m+1)}{2}\right)+2 \cdot \cos \left(\frac{\pi \cdot(m+1)}{2}\right)+5\right)+1+k \cdot 5 \cdot 2^{m+3} .
$$

It follows that the amount of bases $a$ such that $V(a)=m+2$ is proportional to the reciprocal of the (unique) period $k \cdot 5 \cdot 2^{m+3}$.

We proceed by induction on $m$ in order to prove that (8) is true for any $v \in \mathbb{N}: v=m+2$. 
We prove the base case. $m=0 \Rightarrow 2=V(a) \Leftrightarrow V\left(a+k \cdot 5 \cdot 2^{3}\right)=2$, while $m=1 \Rightarrow 3=V(a) \Leftrightarrow V\left(a+k \cdot 5 \cdot 2^{4}\right)=3$, for any $a$. Hence,

$$
\lim _{N \rightarrow+\infty}\left(\frac{\sum_{n=0}^{N} \delta_{V\left(a_{n}\right), 2}}{\sum_{n=0}^{N} \delta_{V\left(a_{n}\right), 3}}\right)=\frac{k \cdot 5 \cdot 2^{4}}{k \cdot 5 \cdot 2^{3}}=2
$$

is true (since $N \rightarrow+\infty$ guarantees that $k$ is always greater than zero).

Now, we define the induction step. Let $t \in \mathbb{N}_{0}$ be given and suppose

$$
\lim _{N \rightarrow+\infty}\left(\frac{\sum_{n=0}^{N} \delta_{V\left(a_{n}\right), m+2}}{\sum_{n=0}^{N} \delta_{V\left(a_{n}\right), m+3}}\right)=2
$$

is true for $m=t$. Then, $2+t=V(a) \Leftrightarrow V\left(a+k \cdot 5 \cdot 2^{3+t}\right)=2+t$, while $t+1=V(a) \Leftrightarrow$ $V\left(a+k \cdot 5 \cdot 2^{4+t}\right)=t+1$. Hence,

$$
\lim _{N \rightarrow+\infty}\left(\frac{\sum_{n=0}^{N} \delta_{V\left(a_{n}\right), t}}{\sum_{n=0}^{N} \delta_{V\left(a_{n}\right), t+1}}\right)=\frac{k \cdot 5 \cdot 2^{2+t+1}}{k \cdot 5 \cdot 2^{2+t}}=2
$$

is also true.

Thus (8) holds for $m=t+1$, and this concludes the proof of the inductive step.

Therefore, (8) is true for any $v \in \mathbb{N}-\{1,2\}$.

Corollary 2. Let $b \in \mathbb{N}: b \geq 3$ be the hyperexponent of the tetration ${ }^{b} a$. $\forall v \in \mathbb{N}, \exists a$, not a multiple of 10 , such that $V(a)=v$.

Proof. Theorem 1 implies the existence of infinite bases of the form

$$
a=2^{m+2} \cdot\left(4 \cdot \sin \left(\frac{\pi \cdot(m+1)}{2}\right)-2 \cdot \cos \left(\frac{\pi \cdot(m+1)}{2}\right)+5\right)-1+k \cdot 5 \cdot 2^{m+3}
$$

such that $V(a)=2+m$, for any $m \in \mathbb{N}_{0}$.

In order to complete the proof, we need to show the existence of a base $\breve{a}$ such that $V(\breve{a})=1$, since $V(1)=0$ follows from the definition stated in Section 3. Lemma 1 gives us $\breve{a}=3 \Rightarrow V(\breve{a})=1$ for any $b \geq 2$.

Therefore,

$$
v=\left\{V(1) \cup V(3) \cup V\left(2^{m+2} \cdot\left(4 \cdot \sin \left(\frac{\pi \cdot(m+1)}{2}\right)-2 \cdot \cos \left(\frac{\pi \cdot(m+1)}{2}\right)+5\right)-1\right)\right\}
$$

covers any natural number, including zero.

Another (constructive) way to prove Corollary 2 would have been to verify that, for any $n$-digits long base $a:=10^{n}-1, V(a)=n(\forall b)$. Moreover, we infer that, $\forall k \in \mathbb{N}_{0}$, $V\left(a: a=\left(10^{n}-1\right)^{\left(10^{k}\right)}\right)=V\left(\left(10^{n}-1\right)+k\right)$, (see [6, pp. 25-26]).

Finally, it is trivial to note that from Corollary 1 and the proof of Corollary 2 it naturally follows that

$$
\lim _{N \rightarrow \infty}\left(\frac{\sum_{n=0}^{N} \delta_{V\left(a_{n}\right), v}}{\sum_{n=0}^{N} \delta_{V\left(a_{n}\right), v+c}}\right)=2^{c+1}
$$

for any $c \in \mathbb{N}_{0}$. 


\section{Periods of the cycles of $a$ such that $V(a)$ is given}

The question that we wish to answer in this section is: "Let $V(a)$ be unique for any given base of the tetration ${ }^{b} a$ (assume for simplicity that $b \in \mathbb{N}: b \geq(a+1) \wedge a \in \mathbb{N}-\{1\}: a \not \equiv$ $0(\bmod 10))$, is it possible to identify the function $\mathcal{P}: \mathbb{Z}_{+} \rightarrow \mathbb{Z}_{+}$defined as

$\mathcal{P}(V(a))=\min \left(P \in \mathbb{N}-\{0\}: V(a)=V(a+k \cdot P), \forall k \in \mathbb{N}_{0} \wedge \forall a \in \mathbb{N}-\{1\}: a \not \equiv 0(\bmod 10)\right)$

(e.g., $a=7 \Rightarrow \mathcal{P}(V(7)=2)=\mathcal{P}(2)=1000$ by Hypothesis 2$)$ ?"”.

Corollary 2 assures us that $\mathcal{P}(V(a))$ is well-defined $\forall V(a) \in \mathbb{N}-\{0\}$. Moreover, we claim that

$$
\mathcal{P}(V(a))=\left\{\begin{array}{ll}
25 & \text { iff } V(a)=1 \\
10^{V(a)+1} & \text { iff } V(a) \geq 2
\end{array} .\right.
$$

In order to show the validity of (10), let us firstly solve a weaker version of the problem above, introducing the additional condition that any base of the integer tetration ${ }^{b} a$ must belong to the set

$$
\mathcal{M}=\left\{\left(a: a(\bmod 25) \in \mathbb{C}^{C}\right) \cup(a: a \equiv 5(\bmod 10))\right\},
$$

where $\mathbb{C}^{C}=\{2,3,4,6,8,9,11,12,13,14,16,17,19,21,22,23\}$ as stated in Hypothesis 1. Under the aforementioned additional constraint, we derive the following lemma.

Lemma 2. Let $b \in \mathbb{N}: b \geq(a+1)$ and let every base of the tetration ${ }^{b} a$ belong to the set $\mathcal{M}=\left\{a:\left(a(\bmod 25) \in \mathbb{C}^{\mathrm{C}} \vee a \equiv 5(\bmod 10)\right)\right\}$. For every $v \in \mathbb{N}-\{0\}: v=V(a)$, the function

$$
\mathcal{P}^{\prime}(v):=\min (P \in \mathbb{N}-\{0\}: v=V((a \in \mathcal{M})+k \cdot P), \forall k \in \mathbb{N}) \cup \mathcal{P}(V(a)=1)
$$

can be rewritten as

$$
\mathcal{P}^{\prime}(v)=\mathcal{P}^{\prime}(V(a \in \mathcal{M}))= \begin{cases}25 & \text { iff } v=1 \\ 2^{v} \cdot 10 & \text { iff } v \geq 2\end{cases}
$$

Proof (assuming Hypothesis 1): It is trivial to point out that Hypothesis 1 covers the case $\mathcal{P}^{\prime}(V(a \in \mathcal{M}))=25$ iff $v=1$. Thus, $a(\bmod 25) \in \mathbb{C}^{C} \Leftrightarrow V(a)=1 \Leftrightarrow \mathcal{P}^{\prime}(V(a))=25$.

If $a: a \equiv 5(\bmod 10)$, then $V(a)$ is always greater or equal to 2 .

By Theorem 1, we have $V(a)=2+m \Leftrightarrow a=\left(x_{m} \vee y_{m}\right)+k \cdot 10 \cdot 2^{m+2}, \forall m \in \mathbb{N}_{0}$.

Therefore, the fundamental period of $x_{m}$ is the same as the fundamental period of $y_{m}$, and it is equal to $10 \cdot 2^{m+2}$. Since, for any $k \in \mathbb{N}_{0}, \quad a: a \equiv 5(\bmod 10) \Rightarrow$ $a=\left(x_{m} \vee y_{m}\right)+k \cdot 10 \cdot 2^{m+2}$, and considering that $v=m+2$, we deduce that (11) is true.

Now, let us examine the result that follows from Lemma 2.

Let the fundamental period of $v_{5}:=V(a: a \equiv 5(\bmod 10))$ be $P_{5}\left(v_{5}\right)=5 \cdot 2^{v+1}$.

The fundamental period of $v_{\{2,4,6,8\}}:=V(a: a(\bmod 10) \in\{2,4,6,8\})$ would most likely be $P_{\{2,4,6,8\}}\left(v_{\{2,4,6,8\}}\right)=5 \cdot 10^{v}$ (see [6, pp. 23-24]). Thus, considering any base $a \not \equiv 0(\bmod 10)$ such that $v_{\{2,3,4,5,6,7,8,9\}}:=V(a: a(\bmod 10) \in\{2,3,4,5,6,7,8,9\}), P_{\{2,3,4,5,6,7,8,9\}}\left(v_{\{2,3,4,5,6,7,8,9\}}\right) \geq$ $\operatorname{gcd}\left(5 \cdot 2^{v+1}, 2^{v} \cdot 5^{v+1}\right)=10^{v+1}=P_{1}(V(a: a \equiv 1(\bmod 10)))$.

Hence, $\exists i \in \mathbb{N}-\{0\}$ such that $P(V(a: a(\bmod 10) \in\{1,2,3,4,5,6,7,8,9\}))=i \cdot 10^{v+1}$, and we conjecture that, $\forall V(a) \in \mathbb{N}-\{0,1\}, i=1$. 
Theorem 2. Let $V(a)$ be strictly greater than 1 . If $\mathcal{P}(V(a)):=\min (P \in \mathbb{N}-\{0\}: V(a)=$ $V(a+k \cdot P), \forall k \in \mathbb{N} \wedge \forall a \in \mathbb{N}-\{1\}: a \not \equiv 0(\bmod 10))$, then $\mathcal{P}(V(a)) \in \mathbb{N} \Leftrightarrow \mathcal{P}(V(a))=$ $10 \cdot i \cdot \mathcal{P}(V(a)-1)$, where $i \in \mathbb{N}-\{0\}$.

Proof (assuming Hypothesis 2). If $a$ is the base of the integer tetration ${ }^{b} a$ (for simplicity assume $b \geq a)$, then $V(a, b)=V(a)$ for any $a \in \mathbb{N}-\{1,2\}: a \not \equiv 0(\bmod 10)$ (by Property 1$)$, while $V(2)=1 \forall b \in \mathbb{N}: b \geq 3$ (see [1, p. 148]).

We prove Theorem 2 by induction on $V(a)$ : " $\forall V(a) \geq 2, \mathcal{P}(V(a))=10 \cdot i \cdot \mathcal{P}(V(a)-1)$, where $i \in \mathbb{N}-\{0\}$ ".

Let us start with the base case, so $V(a)=2$. Assuming Hypothesis $2, \mathcal{P}(V(a)=2)=$ $\mathcal{P}(1) \cdot \mathcal{P}^{\prime}(2)=4 \cdot 10 \cdot \mathcal{P}(1)$ by Lemma $2($ see $(11))$, and $i=4$.

In order to prove the inductive step, let $n \in \mathbb{N}-\{0,1\}$ be given and suppose Theorem 2 holds for $V(a)=n$. It is easy to verify that, $\forall c \in \mathbb{N}$ such that $c \not \equiv 0(\bmod 10)$, $V\left(c \cdot 10^{n}+1\right)=n[6$, p. 21].

In particular, we have $V\left(10^{n}+1\right)=V\left(2 \cdot 10^{n}+1\right)=\ldots=V\left(c \cdot 10^{n}+1\right)=n$, and $n<V\left(a: a \equiv 1\left(\bmod 10^{n+1}\right)\right)$.

It follows that, $\mathcal{P}(n+1)=\mathcal{P}\left(V\left(c \cdot 10^{n+1}+1\right)\right)=j \cdot \mathcal{P}(n)$, where $j \in \mathbb{N}-\{0\}$. In fact, for any $n \geq 2$, if $\mathcal{P}(V(a)) \in \mathbb{N}$, then $\mathcal{P}(n+1)$ is a multiple of $\mathcal{P}(n)$ (including the case $j=1 \Rightarrow \mathcal{P}(n+1)=\mathcal{P}(n))$, by definition (see (9)).

Thus, we need to prove that $j$ is a multiple of 10 .

For this purpose, we observe that, by definition (9), $\nexists a: V(a+j \cdot \mathcal{P}(V(a))) \neq V(a)$. We observe also that $V\left(a: a \equiv 1\left(\bmod 10^{n+2}\right)\right)>n+1$. Now, let $c \not \equiv 0(\bmod 10)$ as stated before.

Then, given $j \in \mathbb{N}-\{0\}, \forall n \in \mathbb{N}-\{0,1\}, \nexists c \in \mathbb{N}$ such that

$$
\begin{aligned}
c \cdot 10^{n+1}+1+\mathcal{P}(V(a)+1) & \equiv 1\left(\bmod 10^{n+2}\right) \\
\Rightarrow c \cdot 10^{n+1}+1+j \cdot \mathcal{P}(V(a)) & \equiv 1\left(\bmod 10^{n+2}\right) \\
\Rightarrow c \cdot 10^{n+1}+j \cdot \mathcal{P}\left(c \cdot 10^{n}+1\right) & \equiv 0\left(\bmod 10^{n+2}\right) .
\end{aligned}
$$

Hence, $c+j$ cannot be a multiple of 10 . Since $c \not \equiv 0(\bmod 10)$ by hypothesis, it follows that $j \equiv 0(\bmod 10)$. Otherwise, $\forall j \in \mathbb{N}: j \not \equiv 0(\bmod 10), \exists c \not \equiv 0(\bmod 10)$ such that $(12)$ is true, which is a contradiction (e.g., if $n=2, c \cdot 10^{3}+j \cdot 10^{3} \equiv 0\left(\bmod 10^{4}\right) \Rightarrow c=10-j$ satisfies (12)). Thus $j=i \cdot 10$, where $i \in \mathbb{N}-\{0\}$. This implies that $\mathcal{P}(V(a)+1)=i \cdot 10 \cdot \mathcal{P}(V(a))$.

Therefore, Theorem 2 holds for $V(a)=n+1$, and the proof of the inductive step is complete. By the principle of induction, Theorem 2 is true for every $V(a) \in \mathbb{N}: V(a) \geq 2$.

\section{Conclusion}

Assuming Property 1, we have shown the laws that describe the congruence speed of any base $a$ such that $a \equiv 5(\bmod 10)($ see Theorem 1$)$.

In general, if $a \in \mathbb{N}-\{1\}: a \not \equiv 0(\bmod 10)$, then all the bases of the tetration ${ }^{b} a$ form a set of periodic sequences modulo multiples of 25 for any $V(a)=$ constant, and we claim that the function which maps the fundamental periods is given by (10). 
An interesting question to be answered in conclusion would be: Let $a \in \mathbb{N}$ : $a \not \equiv 0(\bmod 10)$, and assume $b \geq a_{j}$, where $a_{j}:=\min (a: V(a)=j, \forall j \in \mathbb{N}-\{0,1\})$ : is $a_{j} \equiv 5(\bmod 10)$ for any $j$ ?

We are persuaded that the answer is affirmative (Table 1 shows that it is true for any $j<9$ ), but the inference that $\nexists a_{j} \neq \min \left(2^{j+2} \cdot\left(2 \cdot \cos \left(\frac{\pi \cdot(j+1)}{2}\right)-4 \cdot \sin \left(\frac{\pi \cdot(j+1)}{2}\right)+5\right)+1\right.$, $\left.2^{j+2} \cdot\left(4 \cdot \sin \left(\frac{\pi \cdot(j+1)}{2}\right)-2 \cdot \cos \left(\frac{\pi \cdot(j+1)}{2}\right)+5\right)-1\right)$ needs a mathematical proof.

\section{References}

[1] Andreescu, T., Andrica, D., \& Feng Z. (2006). 104 Number Theory Problems: From the Training of the USA IMO Team. Birkhäuser, Boston.

[2] Daccache, G. (2015). Climbing the ladder of hyper operators: tetration, Mathemathics Stack Exchange, Available online at: https://math.blogoverflow.com/ 2015/01/05/climbing-the-ladder-of-hyper-operators-tetration/

[3] Elaqqad (2015). Last Digits of a Tetration, Mathemathics Stack Exchange (Version: 201504-01), Available online at: https: / /math. stackexchange.com/q/1216149

[4] Germain, J. (2009). On the Equation $a^{x} \equiv x(\bmod b)$. Integers: Learning, Memory, and Cognition, 9 (6), 629-638.

[5] Jolly, N. (2008). Constructing the Primitive Roots of Prime Powers, arxiv.org, Available online at: https://arxiv.org/pdf/0809.2139.pdf

[6] Ripà, M. (2011). La strana coda della serie $n^{\wedge} n^{\wedge} \ldots{ }^{\wedge} n$. UNI Service, Trento.

[7] Sloane, N. J. A. (2018). A317824. The Online Encyclopedia of Integer Sequences, Web. 10 Aug. 2018, Available online at: http: //oeis .org/A317824

[8] Sloane, N. J. A. (2018). A317903. The Online Encyclopedia of Integer Sequences. Web. 10 Aug. 2018, Available online at: http://oeis.org/A317903

[9] Sloane, N. J. A. (2018). A317905. The Online Encyclopedia of Integer Sequences. Web. 10 Aug. 2018, Available online at: http: / / oeis.org/A317905

[10] Urroz, J. J., \& Yebra J. L. A. (2009) On the Equation $a^{x} \equiv x\left(\bmod b^{n}\right)$, Journal of Integer Sequences, 8 (8).

[11] User26486, \& Brandt, M. Do the last digits of exponential towers really converge to a fixed sequence? Mathematics Stack Exchange (Version: 22 Feb. 2015), Available online at https://math. stackexchange. com/questions/1159995/do-the-lastdigits-of-exponential-towers-really-converge-to-a-fixedsequence

[12] Vladimir, \& Doctor Jacques (2005). Modular Exponentiation, The Math Forum, Available online at: http://mathforum.org/library/drmath/view/51625.html

[13] Yan, X.-Y., Wang, W.-X., Chen, G.-R., \& Shi, D.-H. (2016). Multiplex congruence network of natural numbers, Scientific Reports, 6, Article No. 23714. 